\title{
Extended Forward Sensitivity Analysis for Uncertainty Quantification
}

\author{
NURETH-14
}

Haihua Zhao

Vincent A. Mousseau

September 2011

The INL is a

U.S. Department of Energy

National Laboratory

operated by

Battelle Energy Alliance

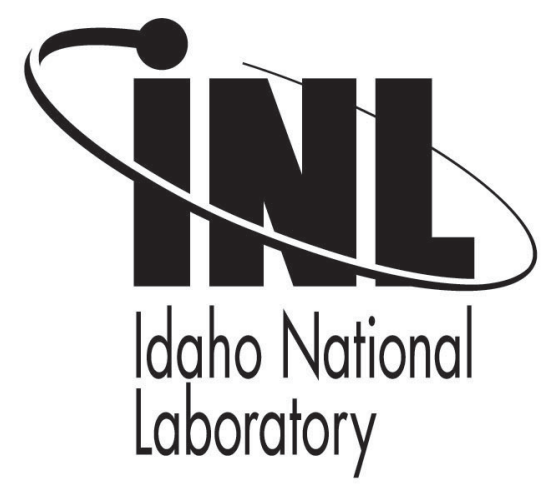

This is a preprint of a paper intended for publication in a journal or proceedings. Since changes may be made before publication, this preprint should not be cited or reproduced without permission of the author. This document was prepared as an account of work sponsored by an agency of the United States Government. Neither the United States Government nor any agency thereof, or any of their employees, makes any warranty, expressed or implied, or assumes any legal liability or responsibility for any third party's use, or the results of such use, of any information, apparatus, product or process disclosed in this report, or represents that its use by such third party would not infringe privately owned rights. The views expressed in this paper are not necessarily those of the United States Government or the sponsoring agency. 


\title{
EXTENDED FORWARD SENSITIVITY ANALYSIS FOR UNCERTAINTY QUANTIFICATION
}

\author{
Haihua Zhao ${ }^{1}$ and Vincent A. Mousseau ${ }^{2}$ \\ ${ }^{1}$ Idaho National Laboratory, Idaho Falls, ID, USA, haihua.zhao@inl.gov \\ 2 Sandia National Laboratories, Albuquerque, NM, USA, vamouss@,sandia.gov
}

\begin{abstract}
This paper presents the extended forward sensitivity analysis as a method to help uncertainty qualification. By including time step and potentially spatial step as special sensitivity parameters, the forward sensitivity method is extended as one method to quantify numerical errors. Note that by integrating local truncation errors over the whole system through the forward sensitivity analysis process, the generated time step and spatial step sensitivity information reflect global numerical errors. The discretization errors can be systematically compared against uncertainties due to other physical parameters. Two well-defined benchmark problems with manufactured solutions are utilized to demonstrate the method.
\end{abstract}

\section{Introduction}

Verification and validation $(\mathrm{V} \& \mathrm{~V})$ are playing more important roles to quantify uncertainties and realize high fidelity simulations in engineering system analysis, such as transients happened in a complex nuclear reactor system. Traditional V\&V in system analysis focused more on the validation part or did not differentiate verification and validation. Progress in $\mathrm{V} \& \mathrm{~V}$ in CFD fields [1] and from broader software engineering fields makes it possible to obtain high confidence in new high fidelity software. For example, order-of-accuracy verification in a 3-D CFD code has been performed through method of manufactured solution (MMS) [2] to verify second order accuracy and identify the existence of any first order errors. Advances in sensitivity analysis techniques [3][4] can be utilized to quantify uncertainties, which is meaningful only after rigorous $\mathrm{V} \& \mathrm{~V}$ has guaranteed that the numerical errors are small.

The traditional approach [5] to uncertainty quantification is based on a "black box" approach. The simulation tool is treated as an unknown signal generator, a distribution of inputs according to assumed probability density functions is sent in and the distribution of the outputs is measured and correlated back to the original input distribution. This approach is usually performed with coarse resolution models in space and time because of the larger number of simulation runs (say, 59) required to resolve the distribution of inputs. Even with coarse resolution models, the total computation cost of this method is still high due to the requirement of many runs.

In contrast to the "black box" method, a more efficient sensitivity approach can take advantage of intimate knowledge of the simulation code. In this approach, equations for the 
propagation of uncertainty are constructed and the sensitivities are solved for as variables in the simulation. This can generate similar sensitivity information as the above "black box" approach with a few runs to cover a large uncertainty region. Because only small numbers of runs are required, those runs can be done with enough accuracy in space and time ensuring that the uncertainty of the physical model is being measured and not simply the numerical error caused by the coarse discretization. Because of the increased efficiency and accuracy of this method, the uncertainty of many physical models can be measured and ordered according to uncertainty scales. Given this quantitative measure of uncertainty scales, one can prioritize the effort of model improvement according to where it will result in the largest reduction of uncertainty.

Two of the most popular sensitivity analysis methods are the forward sensitivity method [4] and the adjoint sensitivity method [6][7]. In the forward sensitivity method, the model is differentiated with respect to each parameter to yield an additional system of the same size as the original one, the result of which is the solution sensitivity. The sensitivity of any output variable can then be directly obtained from these sensitivities by applying the chain rule of differentiation. The forward sensitivity method is most suitable when one needs the sensitivity information of many outputs with respect to relatively few parameters. In the adjoint method, the solution sensitivities need not be computed explicitly. Instead, for each output variable of interest, one forms and solves an additional system, adjoint to the original one, the solution of which can then be used to evaluate the gradient of the output variable with respect to any set of model parameters. The adjoint sensitivity method is more practical than the forward approach when the number of parameters is large and when one needs the sensitivities of only few output variables. However, if one considers numerical errors important, extended forward sensitivity method with time step and spatial step as special parameters, which we present in this paper, can be always applicable even for applications with large set of physical parameters. The forward sensitivity method is straight forward to derive and can handle both nonlinear and transient problems well. Adjoint method requires forming Jacobian matrix through complex mathematical derivation. It stores solutions at each time step, so require huge storage for long transients. Adjoint methods have been successfully applied for linear steady state problems. However, huge challenge exists for transient non-linear problems.

This paper focuses on the forward sensitivity analysis method. Two well-defined benchmark problems are used to show and extend the technique. This work not only applies forward sensitivity analysis method with the strictest verification, but also extends the technique to include time step sensitivity and potentially space step sensitivity as one method to quantify numerical errors. The paper is organized in the following way: section 2 summarizes the basic forward sensitivity analysis theory; section 3 presents the extension to include time step as a special sensitivity parameter in the forward sensitivity analysis; section 4 presents the two benchmark problems - thermal wave problem and nonlinear diffusion problem; and the last section presents the conclusions. 


\section{Forward sensitivity method}

General forward sensitivity theory has been discussed in many references [3][4]. For completeness we summarize the basic ideas below. Consider a nonlinear system of partial differential equations

$$
\frac{\partial \mathbf{Y}}{\partial t}-\mathbf{F}(t, \mathbf{Y}, p)=0
$$

Here $\mathbf{Y}$ is the state vector, $\mathbf{t}$ time, $\mathbf{F}$ the vector of the functions, and $p$ a parameter that the solution depends upon. The parameter sensitivity reflects how much the parameter change affects the solution, or in equation form

$$
\mathbf{s}=\frac{d \mathbf{Y}}{d p}
$$

Here $\mathbf{s}$ is the sensitivity of the solution $\mathbf{Y}$ with respect to the parameter $p$ (we define $\mathrm{s}_{\mathrm{p}}$ as $\mathrm{p}$ parameter sensitivity). By differentiating Eq. 1 with the parameter $p$, the equation for the time evolution of the sensitivity $\mathbf{s}$ can be obtained as:

$$
\frac{\partial \mathbf{s}}{\partial t}=\frac{\partial \mathbf{F}}{\partial \mathbf{Y}} \mathbf{s}+\frac{\partial \mathbf{F}}{\partial p},
$$

Define residual functions:

$$
\begin{gathered}
\mathbf{f}(\mathbf{Y})=\frac{\partial \mathbf{Y}}{\partial t}-\mathbf{F}(t, \mathbf{Y}, p), \\
\mathbf{g}(\mathbf{s})=\frac{\partial \mathbf{s}}{\partial t}-\frac{\partial \mathbf{F}}{\partial \mathbf{Y}} \mathbf{s}-\frac{\partial \mathbf{F}}{\partial p},
\end{gathered}
$$

We will solve the nonlinear system of equations

$$
\left[\begin{array}{c}
\mathbf{f}(\mathbf{Y}) \\
\mathbf{g}(\mathbf{s})
\end{array}\right]=0
$$

using Newton's method

$$
\left[\begin{array}{cc}
\widetilde{\mathbf{J}} & \mathbf{0} \\
\mathbf{0} & \widetilde{\mathbf{J}}
\end{array}\right]\left[\begin{array}{c}
\boldsymbol{\delta} \mathbf{Y} \\
\boldsymbol{\delta} \mathbf{s}
\end{array}\right]=-\left[\begin{array}{c}
\mathbf{f}(\mathbf{Y}) \\
\mathbf{g}(\mathbf{s})
\end{array}\right],
$$

Eq. 7 will be iterated until the residuals of Eq. 6 are small. Here $\widetilde{\mathbf{J}}$ is the normal Jacobian matrix for the physical Eq. 1 and can be directly constructed:

$$
\widetilde{\mathbf{J}}=\frac{\partial \mathbf{f}}{\partial \mathbf{Y}}=\frac{\partial}{\partial \mathbf{Y}}\left(\frac{\partial \mathbf{Y}}{\partial t}\right)-\frac{\partial \mathbf{F}}{\partial \mathbf{Y}},
$$


or can be avoided with the Jacobian free method [4]:

$$
\begin{gathered}
\widetilde{\mathbf{J}} \cdot \mathbf{v} \approx \frac{\mathbf{f}(\mathbf{Y}+\varepsilon \cdot \mathbf{v})-\mathbf{f}(\mathbf{Y})}{\varepsilon}, \\
\frac{\partial \mathbf{F}}{\partial \mathbf{Y}} \mathbf{s}+\frac{\partial \mathbf{F}}{\partial p} \approx \frac{\mathbf{F}(t, \mathbf{Y}+\sigma \cdot \mathbf{s}, p+\sigma)-\mathbf{F}(t, \mathbf{Y}-\sigma \cdot \mathbf{s}, p-\sigma)}{2 \sigma},
\end{gathered}
$$

Where $\varepsilon$ and $\sigma$ are small perturbations and $\mathbf{v}$ a Krylov vector.

Note that the sensitivity equation (3) is linear. It means that less CPU time is needed to solve the sensitivity equation than the physical equation. If the Jacobian matrix is formed during the solution procedure for the physical equation, the matrix can be reused to solve the sensitivity equation. For this type of sensitivity problems, special algorithms could result in magnitude of computation saving than directly solving the physical problem twice to generate the sensitivity information [8][9]. For more general system with parameters,

$$
\mathbf{F}\left(t, \mathbf{Y}, \mathbf{Y}^{\prime}, \mathbf{p}\right)=0, \mathbf{Y}(\mathbf{0})=\mathbf{Y}_{\mathbf{0}} \text {, }
$$

various algorithms are available for different problems [3][4] This paper only focuses on the type of problem as shown in Eq. 1 and will not discuss further on how to take advantage of the linear nature of the sensitivity equation to save computation time.

Once we have solved for $\mathbf{Y}$ and $\mathbf{s}$ we can now plot the solution $\mathbf{Y}$ with uncertainty bars based on an uncertainty range in $p$ defined as $\Delta p$,

$$
\mathbf{Y}_{ \pm} \approx \mathbf{Y} \pm \frac{d \mathbf{Y}}{d p} \Delta p=\mathbf{Y} \pm \mathbf{s} \Delta p
$$

There is an important assumption in using Eq. 12. This analysis requires that the truncation error in the solution method is small so that one is analyzing the physical model and not the numerical error. For this analysis the first step is to verify that the solution is not sensitive to the grid spacing $\Delta x$ or time step $\Delta t$. This can be done through two methods: the conventional time step and spatial step convergence study or taking $\Delta x$ and $\Delta t$ as special sensitivity parameters to calculate sensitivities of the solution with respect to them. The later method could be more efficient than conventional convergence study since they only require one run instead of the series runs required for a convergence study. We will derive the method to calculate $\Delta t$ sensitivity in the next section.

\section{Time step sensitivity}

For the solution sensitivities with respect to the time step and the spatial step, one can not directly derive the sensitivity equations from the original PDEs (Partial Differential Equation) since the time and spatial steps are discrete quantities that depend on the discretization method. Let's consider the time step sensitivity. When we discretize a PDE, the actual 
discrete equation to be solved is the original PDE with added local truncation error (LTE). If we subtract the local truncation error term in the discretized PDE, the modified discretized equation will give us a higher order solution. The LTE is a function of the time step. Therefore, we can derive the time step sensitivity from the modified equation:

$$
\frac{\partial \mathbf{Y}}{\partial t}-\mathbf{F}(t, \mathbf{Y}, p)-\operatorname{LTE}(\Delta t)=0
$$

The forward sensitivity residual function for the time step is,

$$
\mathbf{g}\left(\mathbf{s}_{\Delta t}\right)=\frac{\partial \mathbf{s}_{\Delta t}}{\partial t}-\frac{\partial \mathbf{F}}{\partial \mathbf{Y}} \mathbf{s}_{\Delta t}-\frac{\partial \mathbf{F}_{\mathrm{mod}}}{\partial(\Delta t)}
$$

The corresponding modified $\mathbf{F}$ function for the time step sensitivity problem should be:

$$
\mathbf{F}_{\text {mod }}(t, \mathbf{Y}, p, \Delta t)=\mathbf{F}(t, \mathbf{Y}, p)+\mathbf{L T E}(\Delta t)
$$

The second term in the right hind side of Eq. 14 can be approximated by the following equation:

$$
\frac{\partial \mathbf{F}}{\partial \mathbf{Y}} \mathbf{s}_{\Delta \mathbf{t}} \approx \frac{\mathbf{F}\left(x, t, \mathbf{Y}+\sigma \cdot \mathbf{s}_{\Delta \mathbf{t}}\right)-\mathbf{F}\left(x, t, \mathbf{Y}-\sigma \cdot \mathbf{s}_{\Delta \mathbf{t}}\right)}{2 \sigma}
$$

where $\mathbf{F}$ is the original function in Eq. 1 without adding the local time step truncation term; the third term in the right hind side of Eq. 14 can be analytically derived if the analytical form of LTE is available. Sometimes it is not easy to derive the analytical form of the LTE for complex time integration methods. In such case, we can use a numerical method to derive $\frac{\partial F_{\bmod }}{\partial(\Delta t)}$. The LTE has the following form:

$$
\mathbf{L T E}(\Delta t)=(\Delta t)^{q} \cdot \mathbf{f}_{\mathbf{L T E}}
$$

where $\mathrm{q}$ is the order of the time discretization scheme and $\mathbf{f}_{\mathbf{L T E}}$ a function not depending on $\Delta t$. The LTE can also be approximated by the difference of the current lower order scheme residual and a next higher order scheme residual:

$$
\operatorname{LTE}(\Delta t) \approx \operatorname{res}_{\text {LO }}-\operatorname{res}_{\text {HO }},
$$

where res $_{\mathbf{L O}}$ is the current lower order scheme residual and res $_{\mathrm{HO}}$ a higher order scheme residual. According to Eqs. 17 and 18, we can derive

$$
\frac{\partial \mathbf{F}_{\text {mod }}}{\partial(\Delta t)} \approx-q \frac{\text { res }_{\mathbf{L O}}-\text { res }_{\mathbf{H O}}}{\Delta t},
$$


Within each time step iteration we first converge the physical solution and then we calculate the parameter sensitivities. Therefore by the time we use Eq. 19, the lower order scheme residual is already converged and is very close to zero. So we can further simplify Eq. 19 to

$$
\frac{\partial \mathbf{F}_{\text {mod }}}{\partial(\Delta t)} \approx q \frac{\text { res }_{\mathbf{H O}}}{\Delta t},
$$

Eq. 20 defines a general method which is suitable for any time step schemes. We call this method the residual difference method. For any time step discretization schemes, LTEs can be either analytically derived or $\frac{\partial F_{\bmod }}{\partial(\Delta t)}$ can be calculated with the residual difference method. So the discussed time step sensitivity method is a generic method to consider time step errors. The time step sensitivity here reflects the accumulated time step error instead of LTEs. The accumulated time step error (global error) usually cannot be obtained except for special cases with analytical solutions. The spatial step sensitivity equation can be derived with a similar method.

\section{Examples}

We will use the thermal wave problem [10] and non-linear diffusion problem as two simple examples to show the extended forward sensitivity method. Both problems have analytical solutions for the problems themselves and all the parameter sensitivities so that the numerical algorithms can be accurately verified.

\subsection{Thermal wave problem}

The following is the equation for the thermal wave problem:

$$
\frac{\partial T}{\partial t}-\frac{\partial^{2} T}{\partial x^{2}}=f(T, c, \delta)
$$

for $x \in(-\infty,+\infty)$, and $t \geq 0$. We assume an exact solution which describes a thermal wave moving with a constant velocity c and with a constant wave width $\delta$ :

$$
T(x, t, c, \delta)=\frac{1}{2}\left(1-\tanh \left(\frac{x-c t}{\delta}\right)\right),
$$

In Eq. 21, $f(T, c, \delta)$ is the source term for the manufactured solution, which can be calculated by substituting the assumed solution T (Eq. 22) into the left hind side (LHS) of Eq. 21:

$$
f(T, c, \delta)=\frac{2 T(1-T)(c \delta-2+4 T)}{\delta^{2}},
$$


The reference values for $\mathrm{c}$ and $\delta$ are: $\mathrm{c}=2, \delta=1$. In the numerical analysis, we will limit the computation domain to $[-10,10]$. The boundary conditions and initial condition will be directly derived from the analytical solution. So are for the solution sensitivity with respect to parameter $\mathrm{c}$, and the solution sensitivity with respect to the parameter $\delta$. Figure 1 shows the analytical solution $T(x, t, c, \delta)$.

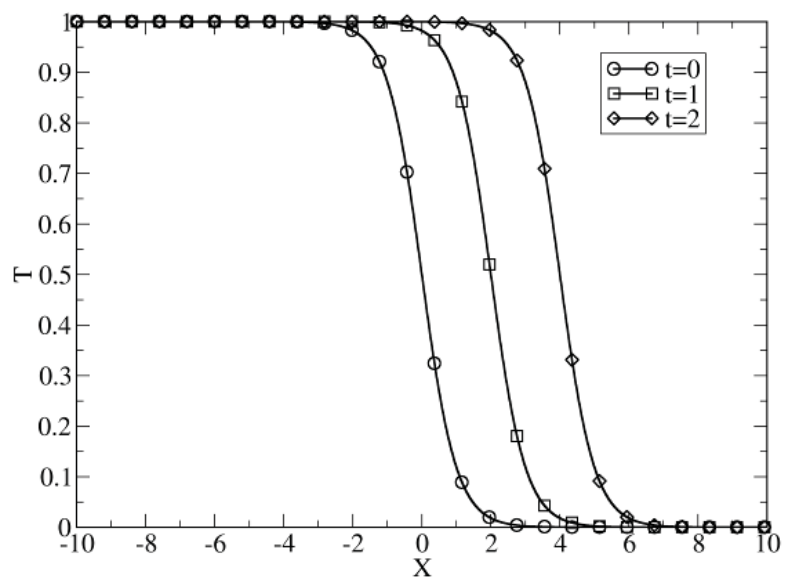

Figure 1 Analytical solution for the thermal wave problem.

Now we will discuss the modified equation for the first order backward Euler scheme (BE) and the associated analytical solution for the time step sensitivity. The local truncation error is

$$
L T E_{t}=\frac{\Delta t}{2} \frac{\partial^{2} T}{\partial t^{2}}
$$

and the modified equation is

$$
\frac{\partial T}{\partial t}-\frac{\partial^{2} T}{\partial x^{2}}-\frac{\Delta t}{2} \frac{\partial^{2} T}{\partial t^{2}}=f(T),
$$

The analytical solution for Eq. 25 with the reference values of $\mathrm{c}=2, \delta=1$ can be derived [11]:

$$
T_{M E A}(x, t, \Delta t)=\frac{1}{2}\left(1-\tanh \left(\frac{x-\frac{2}{\sqrt{1-2 \Delta t}} t}{\frac{1}{\sqrt{1-2 \Delta t}}}\right)\right),
$$

Comparing this equation with Eq. 22, one can see that this solution represents a thermal wave with the width $1 / \sqrt{1-2 \Delta t}$ and speed $2 / \sqrt{1-2 \Delta t}$. The first order temporal error is equivalent 
to simultaneously perturbing " $\mathrm{c}$ " and " $\delta$ " by dividing $\sqrt{1-2 \Delta t}$. According to Eq. 26, we can derive an analytical solution for time step sensitivity for the $1^{\text {st }}$ order backward Euler scheme:

$$
s_{\Delta t}(x, t, \Delta t)=\frac{x\left(1-(\tanh (x \sqrt{1-2 \Delta t}-2 t))^{2}\right)}{2 \sqrt{1-2 \Delta t}},
$$

Eq. 27 will be used as the analytical solution to check the accuracy of the numerical method. Fig. 2 shows $s_{\Delta t}(x, t, \Delta t)$ for three different times with a fixed time step. The solution sensitivity with respect to the time step increases with time and reflects the accumulation of local truncation error over time. The non-intuitive result of nonzero $s_{\Delta t}$ at time zero comes from the dependence on the local truncation error as shown in Eq. 25 and the unsteady state initial condition. If $\mathrm{T}$ is constant before time zero, $\frac{\partial^{2} T}{\partial t^{2}}$ would equal to zero in Eq. 25 and $s_{\Delta t}$ at time zero would be zero.

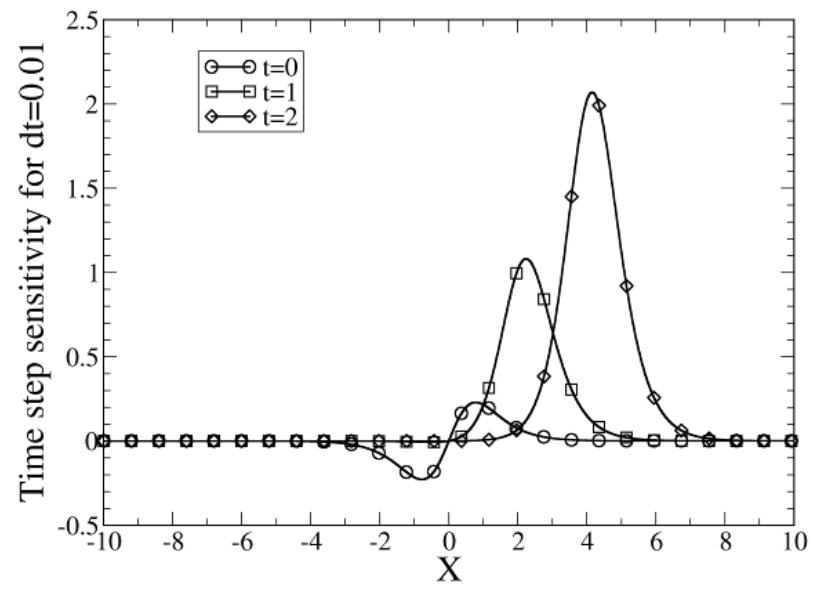

Figure 2 Analytical solution for the sensitivity $\mathrm{S}_{\delta \mathrm{t}}$ for different times.

Both time and spatial steps convergence studies for the physical problem, parameter sensitivities, and time step sensitivity were performed. The numerical results verify correct convergence speeds for all the problems.

The extended forward sensitivity analysis provides a systematic method to evaluate the parameter sensitivity effects on solution, along with time and space convergence information. Fig. 3 compares sensitivity effects from time step, the thermal wave speed, and the thermal wave width parameters for a very small spatial step (1/32) and both a large time step (0.1) and.a small time step (0.001) in the left and right. From the left figure, we can readily notice that the solution is still very sensitive to the time step, which means the solution is not well converged. The uncertainty bar from the time step is much larger than the uncertainty bar from the thermal wave width parameter and is at the same order with the uncertainty bar from 
the thermal wave speed parameter. Therefore, the solution along with the sensitivity analysis is not reliable. One should use a smaller time step in order to obtain accurate solution and sensitivity analysis results. The right figure shows the similar results for a very small time step (0.001) and the same small spatial step (1/32). In this case, the solution is not sensitive to the time step anymore; therefore the solution and sensitivity results are reliable. These comparisons show how important it is to consider numerical errors when performing uncertainty and sensitivity analysis. In conventional Monte Carlo type of uncertainty analysis, one tends to use large time steps and coarse grids in order to perform large amount of calculations, without considering numerical errors. The sensitivity results from such practices often contain large error and even the trends are wrong. Including the time step and the grid size as special sensitivity parameters provides a new method to avoid such pitfalls and improves both accuracy and efficiency.
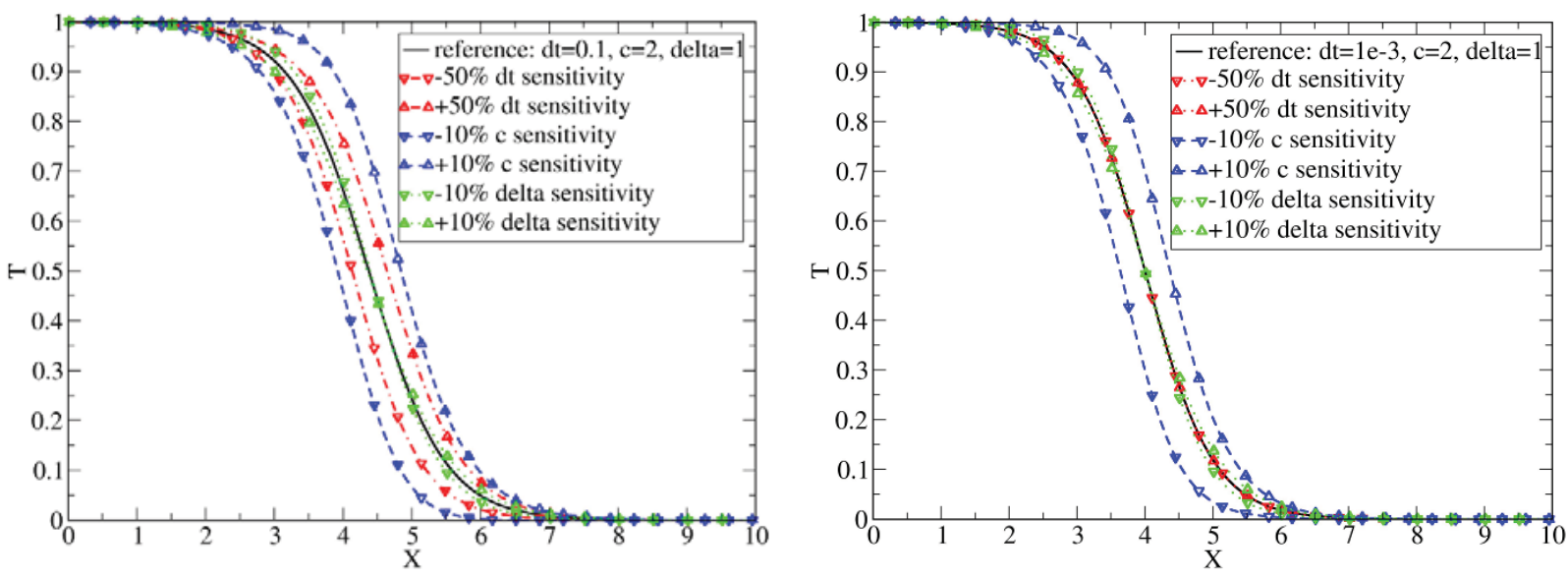

Figure 3 Comparisons of sensitivity effects from time step, wave speed, and wave width for the thermal wave problem, $\mathrm{T}=2$, backward Euler scheme, $\Delta x=1 / 32$

\subsection{Nonlinear diffusion problem}

The following is the equation for the nonlinear diffusion problem:

$$
\frac{\partial T(x, t, \alpha)}{\partial t}-\frac{\partial}{\partial x}\left(T(x, t, \alpha)^{\alpha} \frac{\partial}{\partial x} T(x, t, \alpha)\right)=m m s(x, t, \alpha)
$$

for $x \in[0,1]$, and $t \geq 0$. In Eq. $28 \alpha$ is a constant parameter which does not change with time, $\operatorname{mms}(x, t, \alpha)$ the source term for the manufactured solution depending on the assumed analytical solution, which can be calculated by substituting an assumed solution $T(x, t, \alpha)$ into the LHS. With the analytical solution available, the numerical errors can be accurately measured. The problem meets the boundary conditions: $T(0, t, \alpha)=a, T(1, t, \alpha)=b$, where $a=1$, and $b=0.1$. The initial condition can be obtained from the constructed analytical solution which should be a smooth function with time, space, and the nonlinearity index $\alpha$. Assume the following analytical solution: 


$$
T(x, t, \alpha)=1-0.9 x^{\alpha \cdot t+1}
$$

Substitute Eq. 29 into the LHS of Eq. 28, we obtain:

$$
\begin{aligned}
m m s(x, t, \alpha) & =-0.9 \alpha \cdot x^{\alpha \cdot t+1} \cdot \ln x-0.81 \alpha \cdot\left(1-0.9 x^{\alpha \cdot t+1}\right)^{\alpha-1} \cdot x^{2 \alpha \cdot t} \cdot(\alpha \cdot t+1)^{2} \\
& +0.9 \alpha \cdot\left(1-0.9 x^{\alpha \cdot t+1}\right)^{\alpha} \cdot x^{\alpha \cdot t-1} \cdot t \cdot(\alpha \cdot t+1)
\end{aligned}
$$

This test problem provides the flexibility to adjust the nonlinearity and observe the sensitivity of the solution with the nonlinearity index $\alpha: s_{\alpha}=\frac{d T}{d \alpha}$. Figure 4 shows the analytical solution (Eq. 29) for the case of $\alpha=7$. From the figure, we notice that the nonlinearity increases with time by starting as a linear problem. This problem provides a good test covering both linear and nonlinear problems.

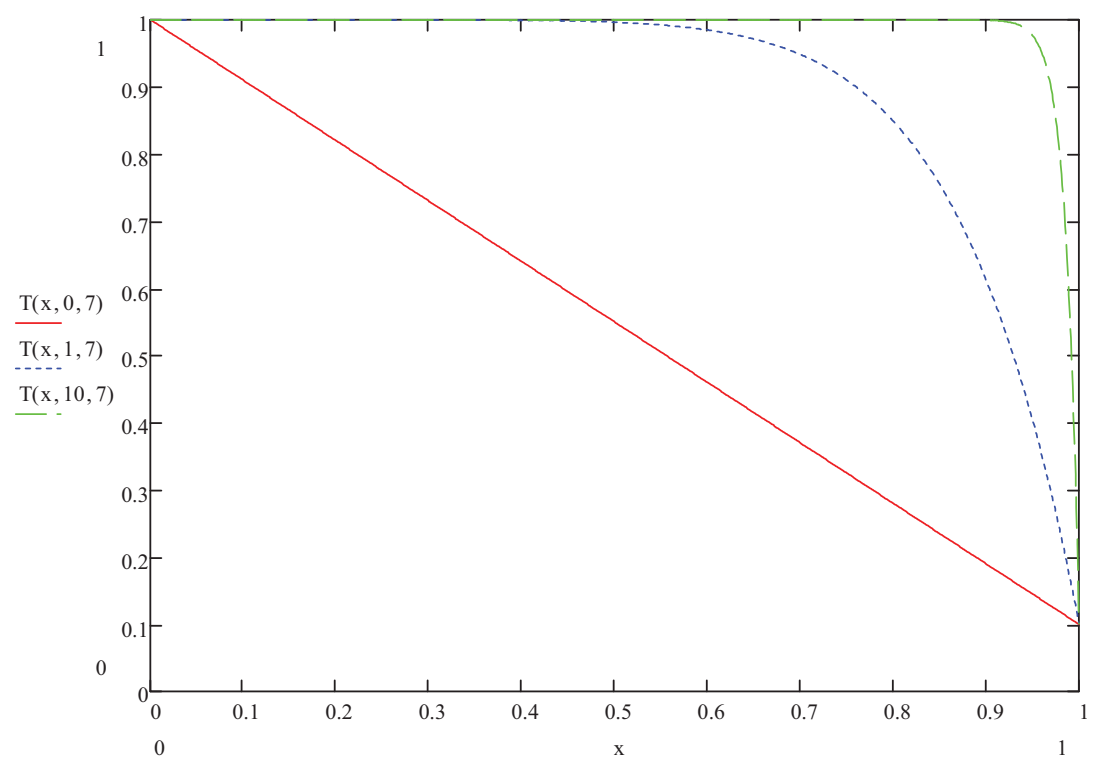

Figure 4 Strong nonlinear diffusion problem analytical solution as function of time.

The local truncation error term for the first order backward Euler scheme is same as Eq. 24. For this problem, we do not have an analytical solution for time step sensitivity $s_{\Delta t}$. Therefore 0 initial condition for $s_{\Delta t}$ is used. The correctness of the calculated $s_{\Delta t}$ is checked according to the convergence rates in space and time and against the results from direct runs with different time steps. 
Figure 5 compares sensitivity effects from time step and $\alpha$ parameter for two time steps $(\Delta t=0.01$, and 0.001$)$ at time equal to 1 . From the left figure, the solution is more sensitive to the physical parameter $\alpha$ than the time step in majority of the computational domain. However, the time step uncertainty bar has same order of width as the physical parameter $\alpha$ uncertainty bar in a small region close to the right boundary, where exists large numerical error. The local numerical error has been correctly captured by the time step sensitivity analysis. Actually, the $-90 \% \Delta \mathrm{t}$ bar corrects the numerical error so successfully that the lower time step uncertainty bar is very close to the analytical solution. The right figure shows similar result at time equal to 1 for a small time step $(\Delta t=0.001)$. In this case, the solution is not sensitive to the time step; therefore the solution and sensitivity results are reliable.

$\mathrm{dx}=1 / 640, \mathrm{dt}=0.01$, time $=1$, Backward Euler

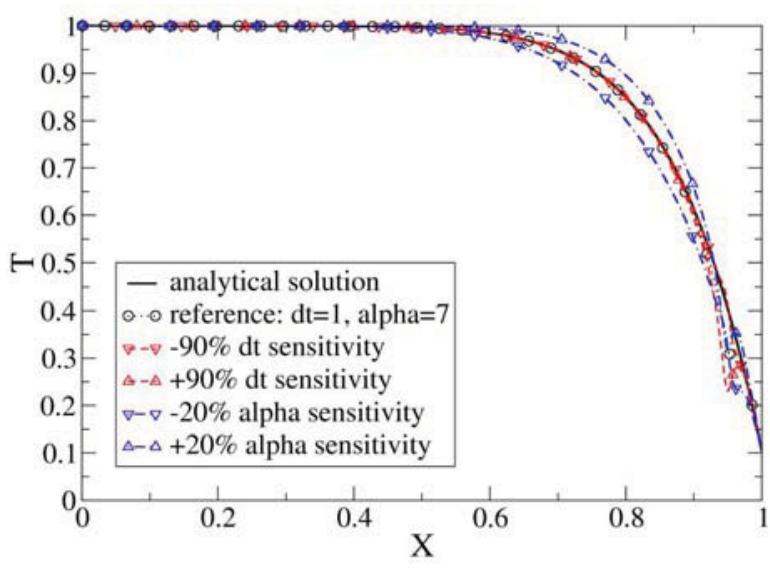

$\mathrm{dx}=1 / 640, \mathrm{dt}=0.001$, time $=1$, Backward Euler

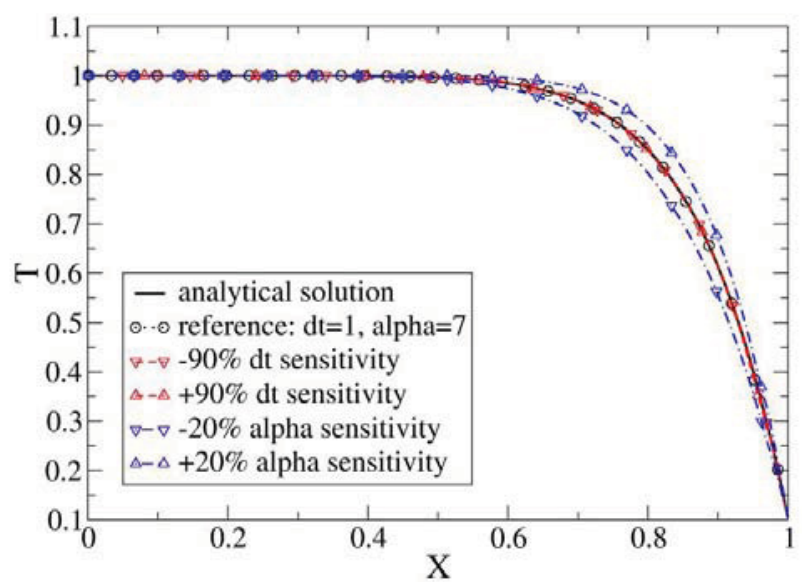

Figure 5 Comparisons of sensitivity effects from time step and $\alpha$ in the strong nonlinear diffusion problem, $\mathrm{t}=1$, backward Euler scheme, $\Delta x=1 / 640$.

\section{Conclusions}

By extending the forward sensitivity method to include time and spatial steps as special parameters, global numerical errors can be quantified against uncertainties from other physical parameters. This extension makes the forward sensitivity method a much more powerful tool to help uncertainty qualification. By knowing the relative sensitivity of time and space steps with other interested physical parameters, simulations are allowed to run at appropriate time and space steps without affecting the confidence to the physical parameter sensitivity results.

The two well defined benchmark problems are utilized to demonstrate the extended forward sensitivity analysis. All the physical solutions, parameter sensitivity solutions, even time step sensitivity, have analytical forms, which allows the verification to be done in the strictest sense. While $\mathrm{V} \& \mathrm{~V}$ has been widely recognized as indispensable to high fidelity simulation, very few of practical works have been pursued to quantify numerical error sensitivity along 
with physical parameter sensitivities. The extended forward sensitivity method can potentially fill the gap.

\section{Acknowledgments}

This work is supported by the U.S. Department of Energy, under DOE Idaho Operations Office Contract DE-AC07-05ID14517. Accordingly, the U.S. Government retains a nonexclusive, royalty-free license to publish or reproduce the published form of this contribution, or allow others to do so, for U.S. Government purposes.

\section{References}

[1] W.L. Oberkampf and T.G. Trucano, "Verification and validation in computational fluids dynamics,” Progress in Aerospace Sciences, Vol. 38, 2002, pp. 209-272.

[2] R.B. Bond, et. al., "Manufactured solution for computational fluid dynamics boundary condition verification," AIAA Journal, Vol. 45, No. 9, 2007, pp. 22242236.

[3] M.D. Tocci, "Sensitivity analysis of large-scale time dependent PDEs," Applied Numerical Mathematics, Vol. 37, 2001, pp. 109-125.

[4] A.C. Hindmarsh, et. al., "SUNDIALS: suite of nonlinear and differential/algebraic equation solvers," ACM Transactions on Mathematical Software, Vol. 31, No. 3, 2005, pp. 363-396.

[5] A. Prosek and B. Mavko, "The state-of-the-art theory and applications of bestestimate plus uncertainty methods," Nuclear Technology, Vol. 158, 2007, pp. 69-79.

[6] D.G. Cacuci and M. Ionescu-Bujor, "Adjoint sensitivity analysis of the RELAP5/MOD3.2 two-fluid thermal-hydraulic code system-I: theory," Nuclear Science and Engineering, Vol. 136, 2000, pp. 59-84.

[7] D.G. Cacuci, Sensitivity and Uncertainty Analysis, Chapman \& Hall/CRC, ISBN 158488-115-1, 2003.

[8] M. Caracotsios and W.E. Stewart, "Sensitivity analysis of initial value problems with mixed ODEs and algebraic equations," Computer\& Chemical Engineering, Vol. 9, No. 4, 1985, pp. 359-365.

[9] J. R. Leis and M.A. Kramer, "Sensitivity analysis of systems of differential and algebraic equations," Computer\& Chemical Engineering, Vol. 9, No. 1, 1985, pp. 93-96.

[10] O. Knio, H. Najm, P. Wyckoff, "A semi-implicit numerical scheme for reacting flow: II. stiff operator-split formulation," Journal of Computational Physics, Vol. 154, 1999, pp. 428-467.

[11] R. Rauenzahn, Los Alamos National Laboratory, personal communication, 2008. 